PHYSICAL REVIEW FLUIDS 1, 041701(R) (2016)

\title{
Minimum-dissipation scalar transport model for large-eddy simulation of turbulent flows
}

\author{
Mahdi Abkar," Hyun J. Bae, and Parviz Moin \\ Center for Turbulence Research, Stanford University, Stanford, California 94305, USA
}

(Received 11 April 2016; published 29 August 2016)

\begin{abstract}
Minimum-dissipation models are a simple alternative to the Smagorinsky-type approaches to parametrize the subfilter turbulent fluxes in large-eddy simulation. A recently derived model of this type for subfilter stress tensor is the anisotropic minimum-dissipation (AMD) model [Rozema et al., Phys. Fluids 27, 085107 (2015)], which has many desirable properties. It is more cost effective than the dynamic Smagorinsky model, it appropriately switches off in laminar and transitional flows, and it is consistent with the exact subfilter stress tensor on both isotropic and anisotropic grids. In this study, an extension of this approach to modeling the subfilter scalar flux is proposed. The performance of the AMD model is tested in the simulation of a high-Reynolds-number rough-wall boundary-layer flow with a constant and uniform surface scalar flux. The simulation results obtained from the AMD model show good agreement with well-established empirical correlations and theoretical predictions of the resolved flow statistics. In particular, the AMD model is capable of accurately predicting the expected surface-layer similarity profiles and power spectra for both velocity and scalar concentration.
\end{abstract}

DOI: 10.1103/PhysRevFluids.1.041701

\section{INTRODUCTION}

Large-eddy simulation (LES) is the state-of-the-art numerical technique for the study of turbulent transport of momentum and scalars in high-Reynolds-number turbulent flows [1,2]. In LES, all turbulent structures larger than the filter scale are resolved and the contribution of the unresolved small-scale eddies is parameterized. A common formalization of LES reduces the range of scales in a simulation by applying a spatial filter to the Navier-Stokes equations as well as the transport equation for scalar concentration. This gives

$$
\begin{gathered}
\partial_{i} \tilde{u}_{i}=0, \quad \partial_{t} \tilde{u}_{i}+\partial_{j}\left(\tilde{u}_{i} \tilde{u}_{j}\right)=-\partial_{i} \tilde{p}+\partial_{j}\left(v \partial_{j} \tilde{u}_{i}\right)-\partial_{j} \tau_{i j}, \\
\partial_{t} \tilde{\theta}+\partial_{i}\left(\tilde{u}_{i} \tilde{\theta}\right)=\partial_{i}\left(D \partial_{i} \tilde{\theta}\right)-\partial_{i} q_{i},
\end{gathered}
$$

where the tilde represents a spatial filtering, $\tau_{i j}=\widetilde{u_{i} u_{j}}-\tilde{u}_{i} \tilde{u}_{j}$ is the subfilter stress tensor, and $q_{i}=\widetilde{u_{i} \theta}-\tilde{u}_{i} \tilde{\theta}$ is the subfilter scalar flux. A common parametrization strategy in LES consists of computing the deviatoric part of the subfilter stress tensor with an eddy-viscosity model

$$
\tau_{i j}^{d}=\tau_{i j}-\frac{1}{3} \delta_{i j} \tau_{k k}=-2 v_{e} \tilde{S}_{i j}
$$

and the subfilter scalar flux with an eddy-diffusivity model

$$
q_{i}=-D_{e} \partial_{i} \tilde{\theta}
$$

where $\tilde{S}_{i j}=\left(\partial_{i} \tilde{u}_{j}+\partial_{j} \tilde{u}_{i}\right) / 2$ is the resolved strain-rate tensor, $v_{e}$ is the eddy viscosity, and $D_{e}$ is the eddy diffusivity. Here $D_{e}$ is related to $v_{e}$ by the subfilter Schmidt number $\mathrm{Sc}_{e}$ such that $D_{e}=v_{e} \mathrm{Sc}_{e}^{-1}$.

One possible approach to define the subfilter eddy viscosity is to use the mixing length approximation, which yields the well-known Smagorinsky model [3]. In this approach the eddy

\footnotetext{
*abkar@stanford.edu
} 
viscosity is modeled as $\nu_{e}=\left(C_{s} \Delta\right)^{2}|\tilde{S}|$, where $|\tilde{S}|=\sqrt{2 \tilde{S}_{i j} \tilde{S}_{i j}}$ and $C_{s}$ is the Smagorinsky coefficient. Likewise, the subfilter eddy diffusivity is modeled as $D_{e}=\Delta^{2} C_{s}^{2} S c_{e}^{-1}|\tilde{S}|$, where $C_{s}^{2} S c_{e}^{-1}$ is a lumped coefficient. In the standard (nondynamic) Smagorinsky model (SSM), the model coefficients are assumed to be constant independent of time, space, and scales. This assumption raises important concerns in the simulation of fluid flows. For instance, the model inappropriately yields eddy dissipation for laminar and transitional flows. In addition, in the context of high-Reynolds-number turbulent boundary layers, in which the viscous sublayer is not resolved, a wall-damping function is needed to account for the local reduction in the subfilter mixing length close to the surface [4]. Hence, in practice, the standard approach would require detailed calibrations of the model coefficients to yield acceptable results [5,6].

The dynamic procedure proposed by Germano et al. [7] and Moin et al. [8] provides an alternative for determining an appropriate local value of the model coefficients without any ad hoc tuning. In this approach, the model coefficients, which are time and space dependent, are obtained by comparing the eddy dissipation at different scales. A key assumption in the dynamic Smagorinsky model (DSM) is that the model coefficients are scale invariant. To account for scale effects in the DSM, Porté-Agel et al. [5,9] introduced the scale-dependent DSM to compute the value of the model coefficients dynamically, while allowing for scale dependence of the coefficients. The DSM has improved subfilter dissipation characteristics with respect to the SSM and switches off for the laminar and transitional flows. However, the major disadvantage of the DSM is its increased computational complexity compared to the SSM and the need for averaging and clipping to attain numerical stability $[10,11]$.

An alternative approach to define the subfilter eddy viscosity is provided by the minimumdissipation models. They is a new class of subfilter models that provide the minimum eddy dissipation required to dissipate the energy of the subfilter scales. The first minimum-dissipation eddy-viscosity model is the QR model, which was developed by Verstappen et al. [12,13] for the isotropic grids. The QR model is based on the invariants of the resolved rate-of-strain tensor and has many desirable practical and theoretical properties compared to the Smagorinsky-type models. In particular, it appropriately switches off for laminar and transitional flows, has low computational complexity compared to the DSM, and is consistent with the exact subfilter stress tensor on isotropic grids. Recently, Rozema et al. [14] proposed a minimum-dissipation model that generalized the desirable properties of the QR model to anisotropic grids. The anisotropic minimum-dissipation (AMD) model has been successfully applied in simulations of decaying grid turbulence on an isotropic grid and in simulations of a temporal mixing layer and turbulent channel flow on anisotropic grids. Since the turbulent scalar transport is a key component of many environmental and engineering turbulent flows, an extension of the AMD approach to modeling the subfilter scalar flux is valuable and is the focus of this study.

In this paper an AMD turbulent eddy-diffusivity model is proposed and, accompanied by the AMD eddy-viscosity model, implemented in the simulation of a high-Reynolds-number rough-wall boundary-layer flow with a constant passive scalar flux at the surface. The derivation of the AMD model is provided in Sec. II. The LES framework and the numerical setup are described in Sec. III. In Sec. IV the LES results are presented. A summary and conclusions are provided in Sec. V.

\section{ANISOTROPIC MINIMUM-DISSIPATION MODEL}

A minimum-dissipation model imposes that the energy of the subfilter scales of the LES solution does not increase:

$$
\partial_{t} \int_{\Omega_{b}} \frac{1}{2} \tilde{u}_{i}^{\prime} \tilde{u}_{i}^{\prime} d x \leqslant 0, \quad \tilde{u}_{i}^{\prime}=\tilde{u}_{i}-\frac{1}{\left|\Omega_{b}\right|} \int_{\Omega_{b}} \tilde{u}_{i} d x
$$

and

$$
\partial_{t} \int_{\Omega_{b}} \frac{1}{2} \tilde{\theta}^{\prime} \tilde{\theta}^{\prime} d x \leqslant 0, \quad \tilde{\theta}^{\prime}=\tilde{\theta}-\frac{1}{\left|\Omega_{b}\right|} \int_{\Omega_{b}} \tilde{\theta} d x,
$$


where $\tilde{u}_{i}^{\prime}$ and $\tilde{\theta}^{\prime}$ are the subfilter scales corresponding to the filter box with domain $\Omega_{b}$ applied to the LES solution and $x \in \Omega_{b}$ [14]. For a rectangular filter box $\Omega_{b}$, with dimensions of $\delta_{1}, \delta_{2}$, and $\delta_{3}$, an upper bound for the subfilter energy can be obtained using the Poincare inequality. There are different formulations of the Poincaré inequality, otherwise known as the Poincaré-Wintinger inequality:

$$
\int_{\Omega_{b}} \frac{1}{2} \tilde{u}_{i}^{\prime} \tilde{u}_{i}^{\prime} d x \leqslant \int_{\Omega_{b}} \frac{1}{2}\left(\hat{\partial}_{i} \tilde{u}_{j}\right)\left(\hat{\partial}_{i} \tilde{u}_{j}\right) d x
$$

and

$$
\int_{\Omega_{b}} \frac{1}{2} \tilde{\theta}^{\prime} \tilde{\theta}^{\prime} d x \leqslant \int_{\Omega_{b}} \frac{1}{2}\left(\hat{\partial}_{i} \tilde{\theta}\right)\left(\hat{\partial}_{i} \tilde{\theta}\right) d x,
$$

where $\hat{\partial}_{i}=C_{i} \delta_{i} \partial_{i}$ is the scaled gradient operator, $C_{i}$ is a modified Poincaré constant, and $\left(\hat{\partial}_{i} \tilde{u}_{j}\right)\left(\hat{\partial}_{i} \tilde{u}_{j}\right) / 2$ and $\left(\hat{\partial}_{i} \tilde{\theta}\right)\left(\hat{\partial}_{i} \tilde{\theta}\right) / 2$ are the scaled velocity and scalar-concentration gradient energy, respectively. The modified Poincaré constant is independent of the size of the filter box and its magnitude only depends on the accuracy of the discretization method (i.e., order of accuracy) for each direction [14].

The Poincare inequality indicates that the energy of the subfilter scales can be confined by imposing a bound on the scaled velocity and scalar-concentration gradient energy. If the eddy viscosity, the eddy diffusivity, and the filter widths are assumed to be constant in the filter box $\Omega_{b}$, then the evolution equations for the scaled velocity and scalar-concentration gradient energy density can be expressed as

$$
\partial_{t}\left[\frac{1}{2}\left(\hat{\partial}_{i} \tilde{u}_{j}\right)\left(\hat{\partial}_{i} \tilde{u}_{j}\right)\right]=-\left(\hat{\partial}_{k} \tilde{u}_{i}\right)\left(\hat{\partial}_{k} \tilde{u}_{j}\right) \tilde{S}_{i j}-\left(v+v_{e}\right) \hat{\partial}_{k}\left(\partial_{i} \tilde{u}_{j}\right) \hat{\partial}_{k}\left(\partial_{i} \tilde{u}_{j}\right)+\partial_{i} f_{i}
$$

and

$$
\partial_{t}\left[\frac{1}{2}\left(\hat{\partial}_{i} \tilde{\theta}\right)\left(\hat{\partial}_{i} \tilde{\theta}\right)\right]=-\left(\hat{\partial}_{k} \tilde{u}_{i}\right)\left(\hat{\partial}_{k} \tilde{\theta}\right) \partial_{i} \tilde{\theta}-\left(D+D_{e}\right) \hat{\partial}_{k}\left(\partial_{i} \tilde{\theta}\right) \hat{\partial}_{k}\left(\partial_{i} \tilde{\theta}\right)+\partial_{i} g_{i}
$$

where $f_{i}$ and $g_{i}$ are the fluxes of velocity and scalar-concentration gradient energy, respectively. Upon spatial integration over the filter box $\Omega_{b}$, the divergence terms $\partial_{i} f_{i}$ and $\partial_{i} g_{i}$ can be rewritten to a boundary integral. Boundary integrals express transport of the velocity and scalar-concentration gradient energy instead of production or dissipation and are therefore ignored in the derivation of the AMD model.

The dissipation at the scale of a filter box can be approximated by application of the Poincaré inequality

$$
\int_{\Omega_{b}}\left(\partial_{i} \tilde{u}_{j}\right)\left(\partial_{i} \tilde{u}_{j}\right) \leqslant \int_{\Omega_{b}} \hat{\partial}_{k}\left(\partial_{i} \tilde{u}_{j}\right) \hat{\partial}_{k}\left(\partial_{i} \tilde{u}_{j}\right) d x
$$

and

$$
\int_{\Omega_{b}}\left(\partial_{i} \tilde{\theta}\right)\left(\partial_{i} \tilde{\theta}\right) \leqslant \int_{\Omega_{b}} \hat{\partial}_{k}\left(\partial_{i} \tilde{\theta}\right) \hat{\partial}_{k}\left(\partial_{i} \tilde{\theta}\right) d x .
$$

As a result, the eddy-viscosity and the eddy-diffusivity models give sufficient eddy dissipation to cancel the production of scaled velocity and scalar-concentration gradient energy, respectively, if the following inequalities hold:

$$
\int_{\Omega_{b}}-\left(\hat{\partial}_{k} \tilde{u}_{i}\right)\left(\hat{\partial}_{k} \tilde{u}_{j}\right) \tilde{S}_{i j} d x \leqslant v_{e} \int_{\Omega_{b}}\left(\partial_{i} \tilde{u}_{j}\right)\left(\partial_{i} \tilde{u}_{j}\right) d x
$$

and

$$
\int_{\Omega_{b}}-\left(\hat{\partial}_{k} \tilde{u}_{i}\right)\left(\hat{\partial}_{k} \tilde{\theta}\right) \partial_{i} \tilde{\theta} d x \leqslant D_{e} \int_{\Omega_{b}}\left(\partial_{i} \tilde{\theta}\right)\left(\partial_{i} \tilde{\theta}\right) d x .
$$


Taking the minimum eddy dissipation that satisfies these conditions gives

$$
v_{e}=\frac{\int_{\Omega_{b}}-\left(\hat{\partial}_{k} \tilde{u}_{i}\right)\left(\hat{\partial}_{k} \tilde{u}_{j}\right) \tilde{S}_{i j} d x}{\int_{\Omega_{b}}\left(\partial_{l} \tilde{u}_{m}\right)\left(\partial_{l} \tilde{u}_{m}\right) d x}
$$

and

$$
D_{e}=\frac{\int_{\Omega_{b}}-\left(\hat{\partial}_{k} \tilde{u}_{i}\right)\left(\hat{\partial}_{k} \tilde{\theta}\right) \partial_{i} \tilde{\theta} d x}{\int_{\Omega_{b}}\left(\partial_{l} \tilde{\theta}\right)\left(\partial_{l} \tilde{\theta}\right) d x} .
$$

By approximating the integrals over the filter box using the midpoint rule for integration, the AMD eddy-viscosity and eddy-diffusivity model can be written as

$$
\nu_{e}=\frac{-\left(\hat{\partial}_{k} \tilde{u}_{i}\right)\left(\hat{\partial}_{k} \tilde{u}_{j}\right) \tilde{S}_{i j}}{\left(\partial_{l} \tilde{u}_{m}\right)\left(\partial_{l} \tilde{u}_{m}\right)}
$$

and

$$
D_{e}=\frac{-\left(\hat{\partial}_{k} \tilde{u}_{i}\right)\left(\hat{\partial}_{k} \tilde{\theta}\right) \partial_{i} \tilde{\theta}}{\left(\partial_{l} \tilde{\theta}\right)\left(\partial_{l} \tilde{\theta}\right)} .
$$

Worth noting here is that the computational complexity of the AMD model is comparable to the computational complexity of the SSM. However, unlike the SSM, the AMD model switches off in the laminar and transitional flows and does not need any ad hoc near-wall treatments. In addition, the AMD model for subfilter turbulent fluxes is consistent with the exact subfilter stress tensor and scalar flux on both isotropic and anisotropic grids. Taylor expansion of the subfilter turbulent fluxes gives

$$
\tau_{i j}=\widetilde{u_{i} u_{j}}-\tilde{u}_{i} \tilde{u}_{j}=\left(\hat{\partial}_{k} \tilde{u}_{i}\right)\left(\hat{\partial}_{k} \tilde{u}_{j}\right)+O\left(\delta x_{i}^{4}\right)
$$

and

$$
q_{i}=\widetilde{u_{i} \theta}-\tilde{u}_{i} \tilde{\theta}=\left(\hat{\partial}_{k} \tilde{u}_{i}\right)\left(\hat{\partial}_{k} \tilde{\theta}\right)+O\left(\delta x_{i}^{4}\right) .
$$

Thus, the eddy dissipation of the exact subfilter turbulent fluxes is

$$
-\tau_{i j} \tilde{S}_{i j}=-\left(\hat{\partial}_{k} \tilde{u}_{i}\right)\left(\hat{\partial}_{k} \tilde{u}_{j}\right) \tilde{S}_{i j}+O\left(\delta x_{i}^{4}\right)
$$

and

$$
-q_{i} \partial_{i} \tilde{\theta}=-\left(\hat{\partial}_{k} \tilde{u}_{i}\right)\left(\hat{\partial}_{k} \tilde{\theta}\right) \partial_{i} \tilde{\theta}+O\left(\delta x_{i}^{4}\right) .
$$

As shown in these equations, the leading-order terms of these expansions are proportional to the terms in the numerator of the AMD model.

In practical applications of the AMD model, the size of the filter box is set equal to a grid cell $\left(\delta_{i}=\Delta_{i}\right)$ and the corresponding Poincare constant is chosen depending on the accuracy of the discretization method for each direction [14]. In particular, the Poincaré constant is $1 / \sqrt{12}$ for a spectral method [12] and is equal to $1 / \sqrt{3}$ for a second-order accurate scheme [15]. In addition, to ensure numerical stability, the eddy viscosity and eddy diffusivity are restricted to have non-negative values to avoid local kinetic energy transfer from unresolved to resolved scales. In particular, the negative values of the eddy viscosity and eddy diffusivity are set to zero at locations where the local eddy dissipation is negative. It should be mentioned that, in the current simulations (and also in the previous implementation of the AMD model in simulation of decaying grid turbulence and turbulent 
channel flow $[14,15])$, the clipping does not lead to anomalies in the instantaneous dynamics of the flow (see the Appendix).

\section{NUMERICAL SIMULATION}

A numerical solution of Eqs. (1) and (2) is obtained by discretizing the computational domain into $N_{x}, N_{y}$, and $N_{z}$ uniformly spaced grid points with the resolution of $\Delta_{x}, \Delta_{y}$, and $\Delta_{z}$ in the streamwise, spanwise, and wall-normal directions, respectively. The horizontal directions are discretized pseudospectrally, while the wall-normal direction is discretized with a second-order accurate method. Hence, in the AMD model, we adopt $C_{x}=C_{y}=1 / \sqrt{12}$ and $C_{z}=1 / \sqrt{3}$ for the modified Poincaré constant. The nonlinear terms are dealiased in Fourier space using the $3 / 2$ rule [16]. The time advancement is based on a second-order-accurate Adams-Bashforth scheme. In the streamwise and spanwise directions, periodic boundary conditions are applied. The upper boundary conditions are a zero-stress zero-flux condition and zero vertical velocity. For the bottom surface, the standard wall-stress formulation based on the Monin-Obukhov similarity theory is employed [17].

To test the performance of the AMD model, a high-Reynolds-number boundary-layer flow with a constant surface scalar flux $\left.q_{3}\right|_{w}=u_{*} \theta_{*}$ is simulated. The wall-normal height of the computational domain is set to $H=1000 \mathrm{~m}$ and the horizontal domain spans are $L_{x}=L_{y}=2 \pi H$. The boundary layer is driven by an imposed uniform pressure gradient $-u_{*}^{2} / H$ in the streamwise direction. Friction velocity $u_{*}$ and aerodynamics surface roughness $z_{0}$ are set to $0.45 \mathrm{~m} / \mathrm{s}$ and $0.1 \mathrm{~m}$, respectively, which is a setup similar to that used in previous studies $[9,18,19]$. The simulations are carried out with resolutions of $48 \times 48 \times 48,72 \times 72 \times 72$, and $96 \times 96 \times 96$.

\section{RESULTS}

Figure 1(a) shows the mean velocity profile obtained from the AMD model with different spatial resolutions. The averaged nondimensional streamwise velocity gradient $\Phi_{M}=\left(\kappa z / u_{*}\right)\left(d\left\langle\tilde{u}_{1}\right\rangle / d z\right)$ is also plotted, in Fig. 1(b), as a function of normalized height. For the homogeneous surface, the mean velocity is expected to be logarithmic in the surface layer, following $U / u_{*}=(1 / \kappa) \ln \left(z / z_{0}\right)$, which occupies the bottom $10 \%-20 \%$ of the simulation domain. Also, the $\Phi_{M}$ predicted by the similarity theory is expected to have a constant value in the surface layer [20]. As can be seen in this figure, the AMD model can accurately predict the log-law profile for the mean streamwise velocity.
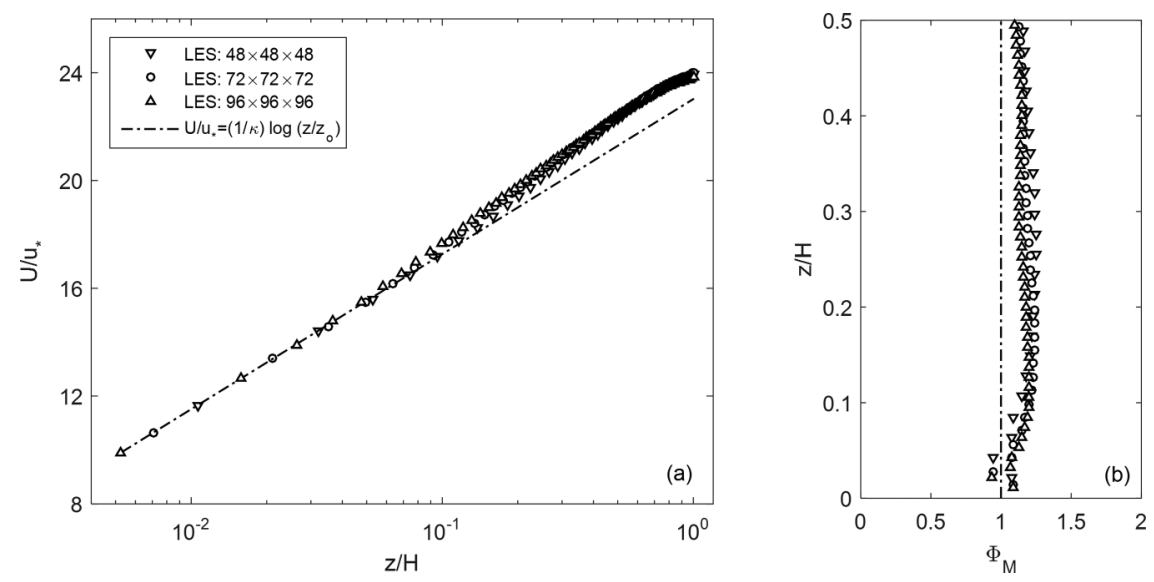

FIG. 1. (a) Normalized streamwise mean velocity profiles in semilogarithmic scale and (b) vertical profiles of the $\Phi_{M}$ function. 
MAHDI ABKAR, HYUN J. BAE, AND PARVIZ MOIN

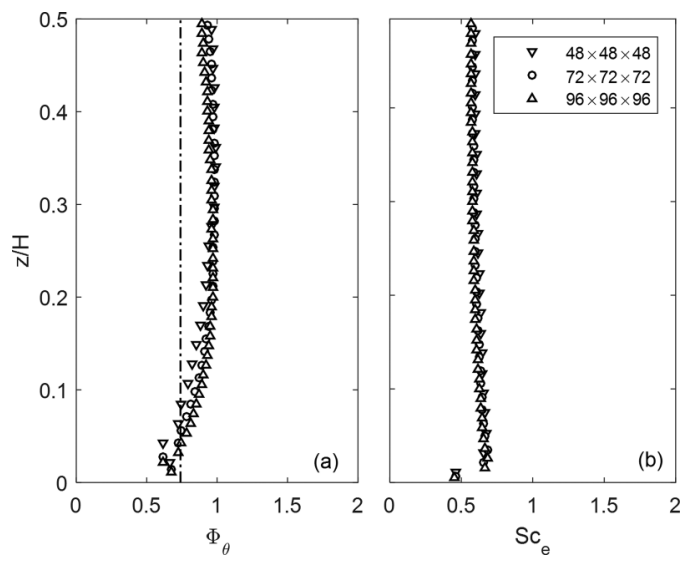

FIG. 2. (a) Vertical profiles of the $\Phi_{\theta}$ function and (b) the subfilter Schmidt number.

For the scalar concentration, since a constant surface flux is imposed as the boundary condition, it is more common to evaluate the averaged nondimensional scalar concentration gradient $\Phi_{\theta}=$ $\left(\kappa z / \theta_{*}\right)(d\langle\tilde{\theta}\rangle / d z)$ instead of the actual value of the scalar concentration. For a passive scalar under neutral stratification, $\Phi_{\theta}$ has been reported to be equal to $0.74[1,20]$ in the surface layer. The vertical profile of $\Phi_{\theta}$ is presented in Fig. 2(a). The values obtained from the AMD model show the expected behavior and compare well with the reported trends using the scale-dependent DSM implementation $[9,18,21]$. The vertical distribution of the subfilter Schmidt number is also shown in Fig. 2(b). This is obtained by dividing the eddy viscosity and eddy diffusivity, followed by averaging in horizontal space and time. In most LESs with a passive scalar, the subfilter Schmidt number is chosen to be a fixed constant value between 0.33 and 0.7 [4,22,23]. As shown in this figure, the obtained value for the subfilter Schmidt number is consistent with those reported in previous studies. In addition, the results show very little sensitivity to the grid resolution.

Figure 3 depicts the total and partial (resolved and subfilter) values of the shear stress and wall-normal scalar flux, respectively. In the absence of viscous effects and under quasi-steady-state conditions, the divergence of the total shear stress must balance the imposed pressure gradient. Also, the divergence of the total scalar flux must balance the rate of change in the scalar concentration. In this study, the boundary-layer flow is driven by a constant streamwise pressure gradient and a
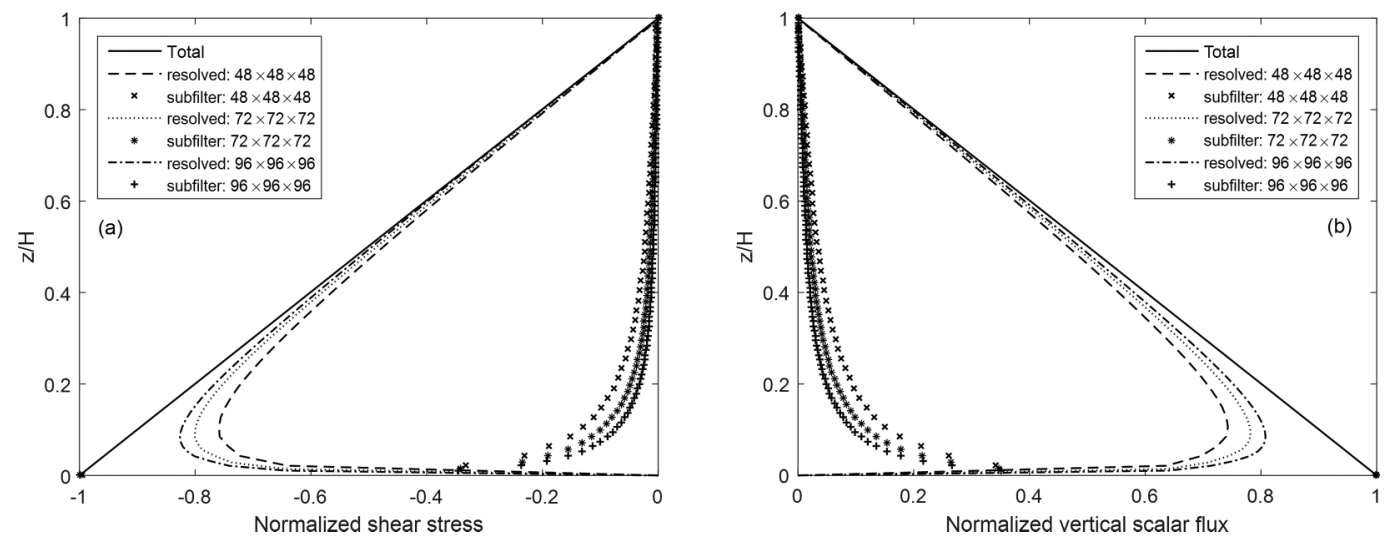

FIG. 3. (a) Vertical profiles of the normalized (by $u_{*}^{2}$ ) total and partial shear stress and (b) the normalized (by $\left.q_{3}\right|_{w}$ ) total and partial wall-normal scalar flux. 
MINIMUM-DISSIPATION SCALAR TRANSPORT MODEL ...
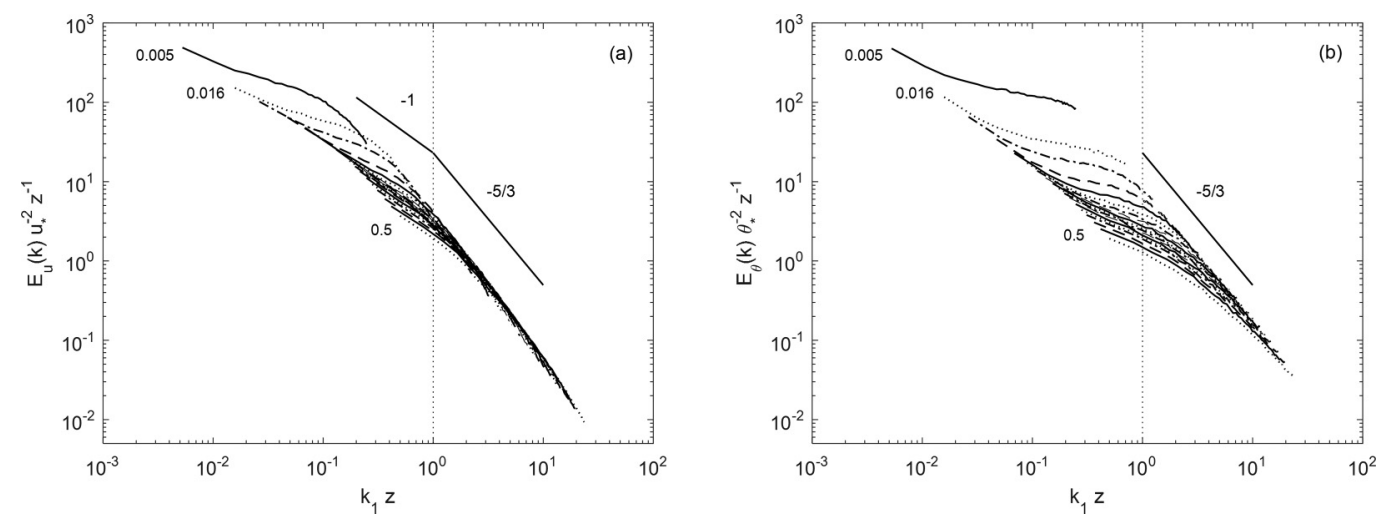

FIG. 4. (a) Normalized resolved streamwise velocity spectra and (b) normalized resolved scalar concentration spectra, obtained from $96^{3}$ simulation. Normalized height $z / H$ increases from 0.005 to 0.5 .

constant scalar flux is imposed at the surface. Therefore, the total shear stress and wall-normal scalar flux are both expected to have linear mean profiles $[5,9,18]$. As shown in these figures, the AMD model reproduces the linear profiles of the total turbulent fluxes, which can serve as a confirmation of stationarity, and momentum and scalar flux conservation of the scheme [19]. The influence of spatial resolution is also observed in these figures. As expected, the contribution of the resolved turbulent fluxes increases as the spatial resolution increases.

In a high-Reynolds-number turbulent boundary-layer flow, it is well known that the velocity and scalar spectra follow the Kolmogorov $-5 / 3$ power law in the inertial subrange $\left(k_{1} z>1\right.$, where $k_{1}$ is the streamwise wave number and $z$ is the distance to the wall) [24,25]. In addition, in the energy-production range $\left(k_{1} z<1\right)$, the velocity spectrum is expected to follow a slope of -1 [26-28]. Figure 4 shows the normalized spectra of the simulated streamwise velocity and scalar concentration, respectively. As demonstrated in these figures, for the small scales $\left(k_{1} z>1\right)$, the normalized spectra show the expected collapse and follow the theoretical inertial subrange scaling with a slope of $-5 / 3$. For scales larger than the distance to the wall $\left(k_{1} z<1\right)$, the slope of the velocity spectra is smaller and close to the expected value of -1 . It is worth mentioning that, as reported in several studies, the SSM yields spectra that decay much faster and have higher slopes at the smallest resolved scale $[5,6,19,23]$. In contrast, the slopes of the power spectra obtained from the DSM are too flat with an unrealistic pileup for the scalar fluctuations at high wave numbers $[9,18]$. The obtained results indicate the ability of the AMD model to accurately predict the transfer of energy from the resolved scales to the subfilter scales at the proper rate.

\section{CONCLUSION}

The AMD model is a simple alternative to the Smagorinsky-type models to parametrize the subfilter turbulent fluxes. It is more cost effective than the DSM, appropriately switches off in laminar and transitional flows, and its subfilter models are consistent with the exact subfilter stress tensor and scalar flux. In this study an AMD model for the subfilter scalar flux was proposed. This model, accompanied by the AMD model for the subfilter stress tensor, was implemented in the simulation of a high-Reynolds-number boundary-layer flow with a constant surface scalar flux. Simulation results obtained from the AMD model reveal good agreement with well-established empirical formulations and theoretical predictions of different flow statistics in a neutral boundary-layer flow. Specifically, the AMD model produces mean velocity and scalar concentration profiles that are in good agreement with similarity theory in the surface layer. In addition, the AMD model is capable of reproducing the expected power-law energy spectra for both velocity and scalar concentration. Future research 
(a)

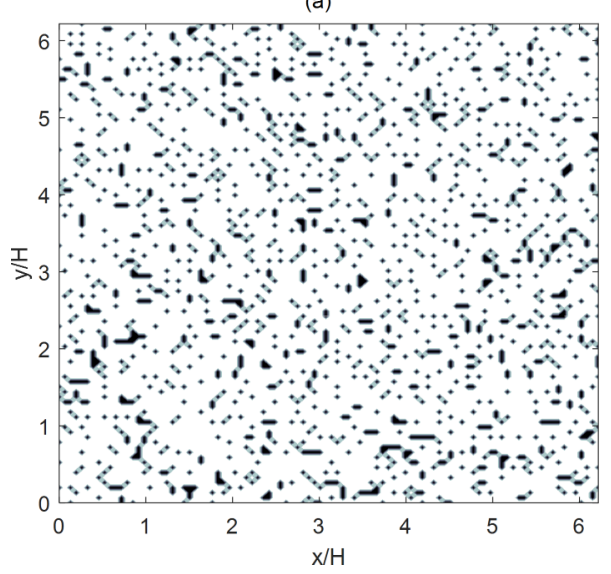

(b)

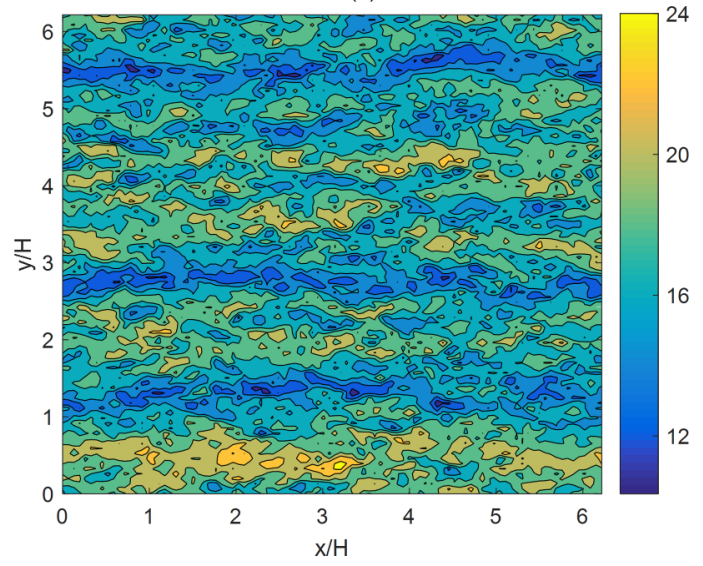

FIG. 5. (a) Instantaneous contours of the locations where $v_{e}=0$ (black diamonds) and (b) the normalized streamwise velocity $\tilde{u}_{1} / u_{*}$ at a height of $z / H=0.1$ from the surface, obtained from $96^{3}$ simulation.

should focus on extending the validation of the AMD model in simulation of high-Reynolds-number boundary-layer flows including the effect of thermal stratification.

\section{ACKNOWLEDGMENTS}

The authors thank Professor John O. Dabiri for his insightful comments on the manuscript. M.A. was supported by the Swiss National Science Foundation.

\section{APPENDIX}

In order to evaluate the effect of clipping procedure in the AMD model on the flow filed, a sample of instantaneous results is shown in Fig. 5. Shown are contour plots of the locations where the eddy viscosity is zero and the streamwise velocity in a horizontal $x-y$ plane at $z / H=0.1$. As shown in this figure, the locations where the clipping occurs are widely distributed throughout the domain and no clear correlation is observed. It should be mentioned that, in the current simulations, the clipping procedure does not cause havoc in computation of the flux derivatives. In particular, the magnitude and the spatial distribution of the flux derivatives obtained from the AMD model are similar to the ones obtained from the DSM (not shown here). In addition, the clipping procedure dose not lead to anomalies in the instantaneous dynamics of the flow. As shown in Fig. 5(b), elongated structures with high-speed and low-speed regions are evident. These coherent streamwise-elongated structures are ubiquitous in turbulent boundary flows and have been repeatedly observed in previous numerical $[29,30]$ and experimental studies [31,32]. This results also show that the locations of zero eddy viscosity are not modulated by the presence of the coherent structures (and vice versa).

[1] R. Stull, An Introduction to Boundary-Layer Meteorology (Kluwer Academic, Dordrecht, 1988).

[2] S. Pope, Turbulent Flows (Cambridge University Press, Cambridge 2000).

[3] J. Smagorinsky, General circulation experiments with the primitive equations, Mon. Weather Rev. 91, 99 (1963).

[4] P. J. Mason and D. J. Thomson, Stochastic backscatter in large-eddy simulations of boundary layers, J. Fluid Mech. 242, 51 (1992).

[5] F. Porté-Agel, C. Meneveau, and M. B. Parlange, A scale-dependent dynamic model for large-eddy simulation: Application to a neutral atmospheric boundary layer, J. Fluid Mech. 415, 261 (2000). 


\section{MINIMUM-DISSIPATION SCALAR TRANSPORT MODEL ...}

[6] E. Bou-Zeid, C. Meneveau, and M. Parlange, A scale-dependent Lagrangian dynamic model for large eddy simulation of complex turbulent flows, Phys. Fluids 17, 025105 (2005).

[7] M. Germano, U. Piomelli, P. Moin, and W. Cabot, A dynamic subgrid-scale eddy viscosity model, Phys. Fluids A 3, 1760 (1991).

[8] P. Moin, K. D. Squires, and S. Lee, A dynamic subgrid-scale model for compressible turbulence and scalar transport, Phys. Fluids A 3, 2746 (1991).

[9] F. Porté-Agel, A scale-dependent dynamic model for scalar transport in large-eddy simulations of the atmospheric boundary layer, Bound.-Lay. Meteorol. 112, 81 (2004).

[10] C. Meneveau, T. S. Lund, and W. H. Cabot, A Lagrangian dynamic subgrid-scale model of turbulence, J. Fluid Mech. 319, 353 (1996).

[11] B. Vreman, B. Geurts, and H. Kuerten, Large-eddy simulation of the turbulent mixing layer, J. Fluid Mech. 339, 357 (1997).

[12] R. Verstappen, S. Bose, J. Lee, H. Choi, and P. Moin, A Dynamic Eddy-Viscosity Model Based on the Invariants of the Rate-of-Strain, Proceedings of the Summer Program, Center for Turbulence Research (Stanford University, Stanford, 2010), pp. 183-192.

[13] R. Verstappen, When does eddy viscosity damp subfilter scales sufficiently? J. Sci. Comput. 49, 94 (2011).

[14] W. Rozema, H. J. Bae, P. Moin, and R. Verstappen, Minimum-dissipation models for large-eddy simulation, Phys. Fluids 27, 085107 (2015).

[15] R. Verstappen, W. Rozema, and H. Bae, Numerical Scale Separation in Large-Eddy Simulation, Proceedings of the Summer Program, Center for Turbulence Research (Stanford University, Stanford, 2014), pp. 417-426.

[16] C. Canuto, M. Hussaini, A. Quarteroni, and T. Zang, Spectral Methods in Fluid Dynamics (Springer, Berlin, 1988).

[17] C. Moeng, A large-eddy simulation model for the study of planetary boundary-layer turbulence, J. Atmos. Sci. 46, 2311 (1984).

[18] R. Stoll and F. Porté-Agel, Dynamic subgrid-scale models for momentum and scalar fluxes in large-eddy simulations of neutrally stratified atmospheric boundary layers over heterogeneous terrain, Water Resour. Res. 42, W01409 (2006).

[19] H. Lu and F. Porté-Agel, A modulated gradient model for scalar transport in large-eddy simulation of the atmospheric boundary layer, Phys. Fluids 25, 015110 (2013).

[20] J. A. Businger, J. C. Wyngaard, Y. Izumi, and E. F. Bradley, Flux-profile relationships in the atmospheric surface layer, J. Atmos. Sci. 28, 181 (1971).

[21] M. Calaf, M. Parlange, and C. Meneveau, Large eddy simulation study of scalar transport in fully developed wind-turbine array boundary layers, Phys. Fluids 23, 126603 (2011).

[22] P. P. Sullivan, J. C. McWilliams, and C.-H. Moeng, A subgrid-scale model for large-eddy simulation of planetary boundary-layer flows, Bound.-Lay. Meteorol. 71, 247 (1994).

[23] A. Andren, A. R. Brown, P. J. Mason, J. Graf, U. Schumann, C.-H. Moeng, and F. T. Nieuwstadt, Large-eddy simulation of a neutrally stratified boundary layer: A comparison of four computer codes, Q. J. R. Meteorol. Soc. 120, 1457 (1994).

[24] S. G. Saddoughi and S. V. Veeravalli, Local isotropy in turbulent boundary layers at high Reynolds number, J. Fluid Mech. 268, 333 (1994).

[25] K. R. Sreenivasan, The passive scalar spectrum and the Obukhov-Corrsin constant, Phys. Fluids 8, 189 (1996).

[26] A. Perry, S. Henbest, and M. Chong, A theoretical and experimental study of wall turbulence, J. Fluid Mech. 165, 163 (1986).

[27] B. A. Kader and A. M. Yaglom, in Turbulence and Coherent Structures, edited by O. Metais and M. Lesieur, Fluid Mechanics and its Applications Vol. 2 (Springer, Berlin, 1991), pp. 387-412.

[28] G. G. Katul, C. R. Chu, M. B. Parlange, J. D. Albertson, and T. A. Ortenburger, Low-wavenumber spectral characteristics of velocity and temperature in the atmospheric surface layer, J. Geophys. Res. 100, 14243 (1995).

[29] X. Wu and P. Moin, Direct numerical simulation of turbulence in a nominally zero-pressure-gradient flat-plate boundary layer, J. Fluid Mech. 630, 5 (2009). 


\section{MAHDI ABKAR, HYUN J. BAE, AND PARVIZ MOIN}

[30] J. Fang and F. Porté-Agel, Large-eddy simulation of very-large-scale motions in the neutrally stratified atmospheric boundary layer, Bound.-Lay. Meteorol. 155, 397 (2015).

[31] C. D. Tomkins and R. J. Adrian, Spanwise structure and scale growth in turbulent boundary layers, J. Fluid Mech. 490, 37 (2003).

[32] N. Hutchins and I. Marusic, Evidence of very long meandering features in the logarithmic region of turbulent boundary layers, J. Fluid Mech. 579, 1 (2007). 